\title{
Capacity and coordination challenges for social assistance in crisis situations
}

\author{
Rachel Slater, Daniela Baur and Ella Haruna
}

\author{
February 2022
}

\section{Theme summary}

The terms 'capacity' and 'coordination' feature consistently in literature on humanitarian cash transfers and social protection. Multiple international agency projects and initiatives seek to build or strengthen both. Yet, while 'capacity' and 'coordination' are commonly used and are frequently identified as deficits that hinder improved programming in crisis situations, there is relatively little understanding of what levels of capacity and coordination exist in fragile settings and of how the dimensions of both might vary in crises compared to more stable and secure situations.
Across the social protection and humanitarian sectors, frameworks for assessing and addressing capacity and coordination are fledgling at best, with little guidance available to those trying to improve capacity and coordination. (Guidance for cash working groups is an exception to this.)

A better understanding of the evidence on capacity and coordination of social assistance during crises and of the main knowledge gaps is key to identifying solutions to overcome capacity and coordination deficits solutions that are fit-for-purpose in situations of protracted crisis.

This thematic brief is a shortened version of a BASIC Research Working Paper. It represents work in progress by the BASIC Research programme. It responds to internal and external peer reviews but not yet to feedback from FCDO.. To explore this research theme in more detail please refer to:

Slater, R.; Haruna, E. and Baur, D. (2022) What is Known About Capacity and Coordination of Social Assistance Programmes in Crisis Situations?, BASIC Research Working Paper 18, Brighton: Institute of Development Studies, DOI: 10.19088/BASIC.2022.018

A full list of the references cited in this brief can be found at the BASIC Research Zotero library: Implemented by
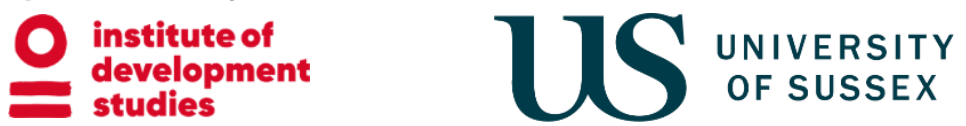

UNIVERSITY OF WOLVERHAMPTON

Better Assistance in Crises (BASIC) Research (funded by UKAid) aims to inform policy and programming on how to help poor and vulnerable people cope better with crises and meet their basic needs through more effective social assistance. All costs related to BASIC Research are covered by the UK Foreign, Commonwealth and Development Office. 


\section{State of the evidence and debate}

\section{Capacity}

- The limited definition and conceptualisation of capacity result in confused and unfocused applications of the concept, dominance of received wisdoms rather than substantive evidence, and largely unhelpful, generalised and sweeping statements about solutions.

- There is little distinction between technical and functional capacities, or between hard and soft skills. Scant attention is paid to the intersection between physical and technical deficits versus political commitments. In general, the literature is simplistic, failing to clarify what or whose capacity we are referring to.

- There are frameworks that offer potential for better understanding capacity in fragile and conflict-affected settings (FCAS):

- A framework that differentiates competence, capability, and performance provides an opportunity in crisis situations to understand how human resources can enable or disable the sustained delivery of programmes. In a protracted crisis setting, otherwise competent staff may have limited capability to navigate challenges in their immediate environment or may perform poorly because of low motivation and being worn down by those challenges.

- Kardhan's (2017) framework incorporates individual, organisational, and institutional capacities; its focus on how capacities are created, utilised, and sustained helps to capture the temporal and dynamic nature of capacities in FCAS.

\section{Capacity describes what a person can do in a standardized, controlled environment. Capability describes what a person can do in his/her daily environment. Performance describes what a person actually does do in his/her daily environment. The person-environment interaction is the discriminating element between capacity, capability, and performance.}

(Holsbeek et al. 2009: 849)

\section{Coordination}

- Coordination is widely acknowledged as important; it is ever-present in the literature, but evidence and analysis about how to improve it are limited in substance. Coordination is rarely defined, rather, it is assumed that audiences and stakeholders are all working to the same (unarticulated) definition. There are sectoral definitions, but these do not clearly translate to the space where humanitarian and social protection overlap.

- So, despite agreement in the literature that effective coordination provides the foundation for humanitarian and social assistance, coordination is also its biggest challenge - from navigating the varied mandates, concerns and priorities of a range of actors, to bringing together distinct funding sources, information systems and modes of reporting (CaLP 2020: 76). Across both humanitarian and social protection sectors, it is often unclear what is being coordinated and through which mechanisms; progress is being made to address this, particularly, for example, the distinction between vertical and horizontal coordination (Smith et al. 2021) (see figure 1). 
Figure 1: Level and domains for coordination of shock response / social protection

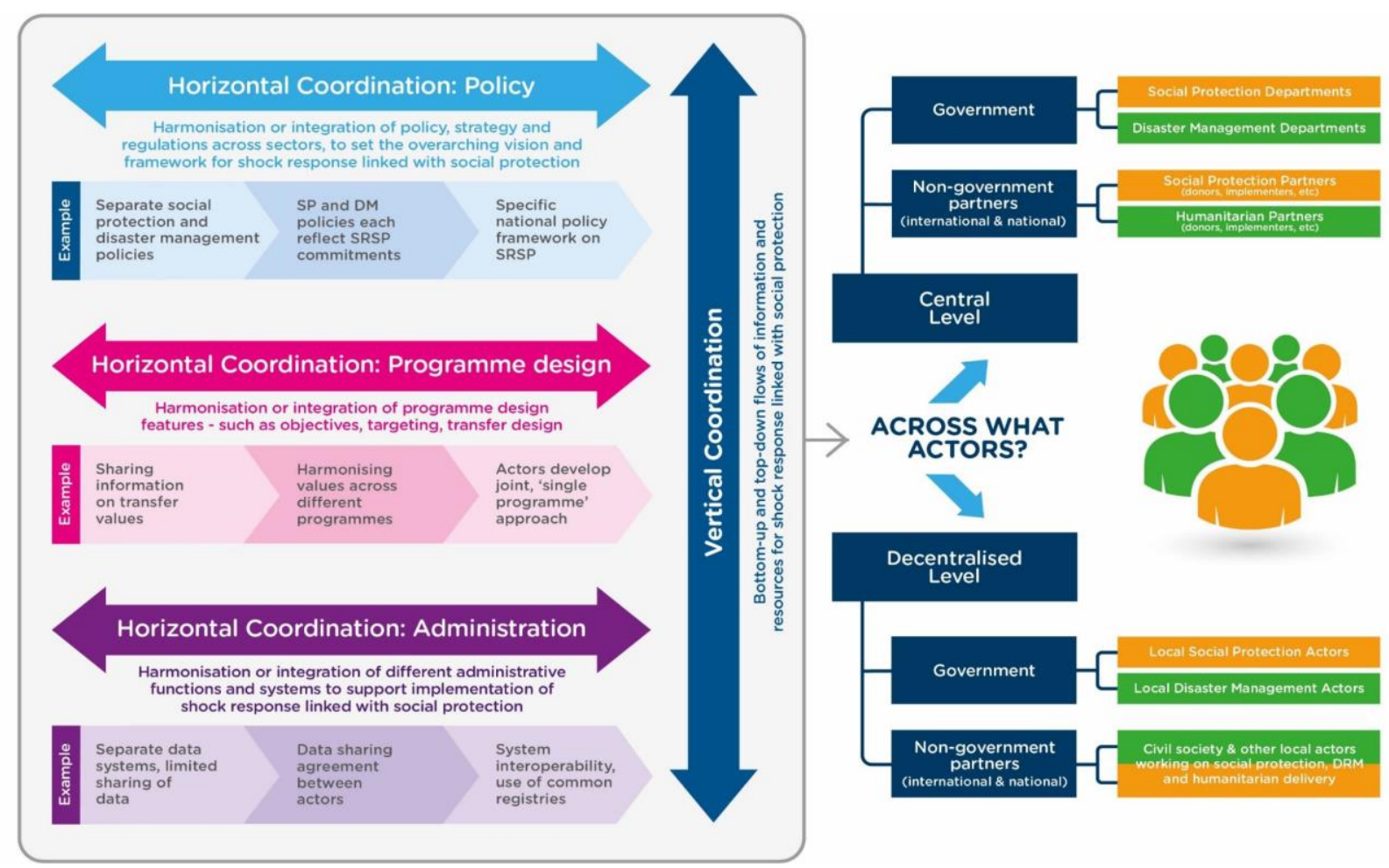

Source: Smith et al. (2021)

\section{Gaps in the evidence}

\section{Capacity}

- While capacity is widely acknowledged as important, substantial evidence and analysis about how to improve it are limited. There is little differentiation of capacity for what and by whom; and of whether 'capacity' concerns the capacity of systems, structures, and/or staff, for example.

- Capacity is often treated narrowly. Capacity deficits for functional or 'soft' skills are frequently overlooked in favour of discussing technical or 'hard' skills. Technical capacities, which include skills associated with subject matter and expertise (knowing how to target, choose payment modalities, establish and maintain registries), receive far more attention than functional capacities, which include skills associated with leadership, negotiation, stakeholder engagement, communication, mainstreaming and coordination.

\section{Coordination}

- Multiple models for coordination of cash exist in the humanitarian sector but there is little robust or systematic analysis on which offer the best prospects for linking humanitarian assistance with social protection and which are likely to result in transformations of social assistance systems that make it more sustainable.

- Coordination is understood in a highly practical, functional, and instrumental way. This perspective has significant limitations, for example, it hinders understanding of where a specific structural / governance / political economy feature is blocking coordination, or recognition that technical solutions may not be enough. There is consequently a key knowledge gap on whether coordination failures in social assistance in crisis situations result from information asymmetries, or principal-agent problems, or collective action failures - or some combination of all three. 


\section{Directions for research}

The roots of capacity challenges in situations of protracted crisis are poorly understood. A framework for understanding capacity that focuses on competencies, capabilities and performance could improve understanding of these roots. It could help to identify solutions that look beyond individual technical competencies and instead encompass individual and organisational functional competencies. Such solutions would tackle behavioural, structural and environmental barriers to social assistance delivery. There is also benefit, in the BASIC Research agenda, in considering the temporal focus on how capacities are created, utilised and sustained in FCAS, in order to better assess in which circumstances governments can best be supported to deliver social assistance. This would minimise situations where humanitarian agencies become the providers of last resort.

- Research could consider: how do competence, capability and performance challenges intersect to undermine social assistance delivery in crisis situations?

To address the lack of established, multi-faceted frameworks for assessing coordination, a forward research agenda focused on action research with organisations delivering social assistance would be useful. This would: (1) build frameworks to better understand coordination challenges and capture their varied elements (such as technical/operational and governance/political/strategic elements); (2) explicitly identify the coordination challenges for overlapping humanitarian and government-led assistance; and (3) work out which coordination solutions lie in this overlap, and which in the respective sectors themselves. There is no disagreement about these overarching coordination challenges when linking humanitarian and social protection programmes - but what is missing is an understanding of how these challenges unfold in specific crises with particular configurations of stakeholders, and with varied social protection programming regimes.

- Research on coordination could consider: what are the parameters of humanitarian-social protection coordination challenges in different crisis contexts, and how can these be overcome?

\section{Acknowledgements and Disclaimer}

This document was developed by the Better Assistance in Crises (BASIC) Research programme. BASIC is implemented by the Institute of Development Studies (IDS), the University of Sussex and the Centre for International Development and Training, funded by UKAid from the UK government. The views expressed in this document are entirely those of the authors and do not necessarily represent views or policies of the UK governments official policies.

(C) IDS copyright 2022. Copyright in the typographical arrangement and design rests with IDS.

This publication (excluding the logos) may be reproduced free of charge in any format or medium, provided that it is reproduced accurately and not used in a misleading context. The material must be acknowledged as IDS copyright with the title and source of the publication specified.

Published by IDS.

DOI: $10.19088 / B A S I C .2022 .030$ 